\title{
La critique de la folie pure chez Montaigne et Shakespeare
}

\section{André Tournon}

\section{OpenEdition}

\section{Journals}

Édition électronique

URL : http://journals.openedition.org/shakespeare/730

DOI : $10.4000 /$ shakespeare. 730

ISSN : 2271-6424

Éditeur

Société Française Shakespeare

Édition imprimée

Date de publication : 1 novembre 2004

Pagination : 277-292

ISBN : 2-9521475-0-7

Référence électronique

André Tournon, "La critique de la folie pure chez Montaigne et Shakespeare », Actes des congrès de la Société française Shakespeare [En ligne], 21 | 2004, mis en ligne le 01 janvier 2007, consulté le 21 décembre 2020. URL : http://journals.openedition.org/shakespeare/730 ; DOI : https://doi.org/ 10.4000/shakespeare.730 


\section{Shakespeare et Montaigne vers un nouvel humanisme}

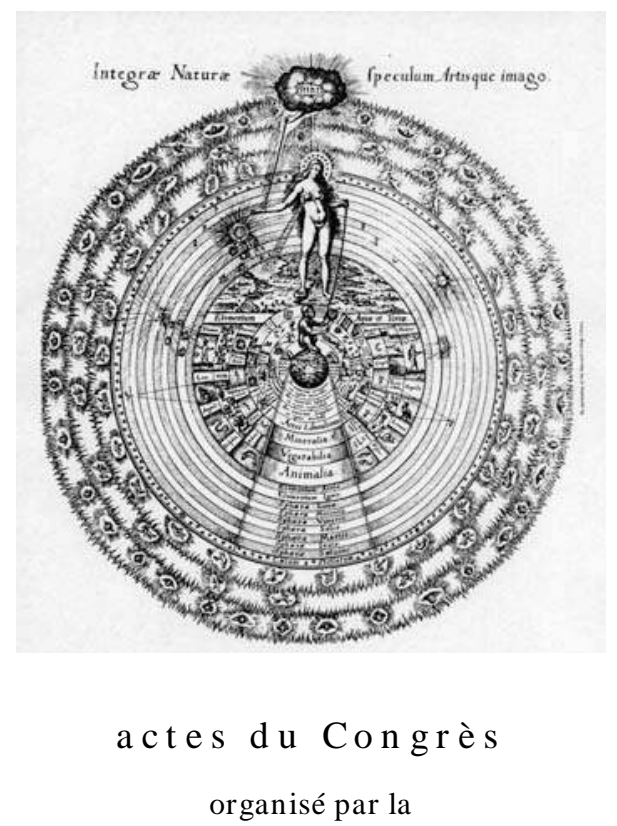

SOCIÉTÉ FR ANÇAISE SH AKESPEARE en collaboration avec la

S OC I É T É I N T E R N A T I O N A L E DES AM I D E M O N A I G N les 13,14 et 15 mars 2003

$$
\begin{gathered}
\text { textes réunis par } \\
\text { Pierre KAPITANIAK } \\
\text { sous la direction de } \\
\text { Jean-Marie MAGUIN }
\end{gathered}
$$




\section{COMITÉ SCIENTIFIQUE :}

Margaret Jones-Davis

Gisèle Venet

Jean-Marie Maguin

Yves Peyré

François Laroque

Pierre Kapitaniak

\section{COUVERTURE :}

Robert Fludd

Utriusque Cosmi Historia (1617-19)

planche 17

conception graphique et logo

Pierre Kapitaniak

\section{(C) 2003 Société Française Shakespeare}

Institut du Monde Anglophone

Université de Paris III - Sorbonne Nouvelle

http:// univ-montp3.fr/SFS/

5 rue de l'École de Médecine

75006 Paris

Diffusion :

AVL DIFFUSION

Parc Euromédecine

34198 MONTPELLIER CEDEX 5

ISBN 2-9521475-0-7

Tous droits de traduction, de reproduction et d'adaptation réservés pour tous les pays 


\title{
LA CRITIQUE DE LA FOLIE PURE CHEZ MONTAIGNE ET SHAKESPEARE
}

\author{
André TOURNON
}

\begin{abstract}
L'intervention portera principalement sur le prétendu éloge de la folie prononcé et immédiatement réfuté dans une addition à l'" A Apologie de Raimond Sebond " (Essais, II, 12, PUF p. 568, Imprimerie Nationale p. 379-380, "N'y a-il point de la hardiesse à la philosophie... "), à confronter avec les différentes formes de "folie" représentées dans King Lear. II s'agira de comprendre : 1) comment la folie " pure ", c'est-à-dire soustraite à la réflexion ironique et aux manœuvres de simulation qui la constitueraient en rôle, est dépouillée des prestiges fantasmatiques dont l'avaient parée la tradition médiévale en ses dernières années, selon Foucault, et les spéculations sur la " mélancolie " dérivées plus récemment des Problemata du pseudo-Aristote ; 2) comment les formes de folie rendues "impures" par la conscience critique décelable en leur jeu peuvent recevoi d'autres fonctions, non moins inquiétantes, en vertu précisément du croisement qu'elles opèrent entre égarement et lucidité. L'intervenant se réserve le droit de s'égarer lui-même, lucidement ou non, à l'écart des axes ainsi tracés.
\end{abstract}

Critique of Pure Folly in Montaigne and Shakespeare This paper will be mainly concerned with the supposed praise of folly stated and immediately refuted in an addition to the "Apology for Raymond Sebond" (Essais, II, 12, Presses Universitaires de France, p. 568, or Imprimerie Nationale edition, p. 379-380, "N'y a-il point de la hardiesse à la philosophie..."), as opposed to the different forms of "folly" represented in King Lear. We will consider: 1) how "pure" folly, that is, taken as being free of irony and the various ruses which would seek to make a role of it, is divested of the fantasmatic prestige which, according to Foucault, both the waning middle ages and speculations on "melancholy" derived subsequently from Pseudo-Aristotle's Problemata attributed to it; 2) how the forms of "folly" made "impure" by the critical conscience discernible in their play can have other uses, which are no less disturbing in that they effect a junction between distraction and lucidity. The author of this paper reserves to himself the right to wander into the paths of distraction, lucidly or otherwise, and away from the themes sketched out above.

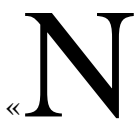

otre sagesse [est] plus folle que la pure folie » avait écrit Montaigne en marge de son «Apologie de Raimond Sebond ${ }^{381}$; ensuite il a raturé l'expression. Celle qu'il lui a substituée est à peine différente, mais n'importe : la trace d'hésitation et de rectification, si ténue soit-elle, indique déjà que sa pensée n'a pas la pureté des déclarations péremptoires que prodiguent les doctrinaires au titre de leur sagesse, et les fous au gré de leurs humeurs. La rature manifeste l'intervention d'un réexamen dans le moment même de l'écriture, et donne à croire qu'à plus vaste échelle la pensée du philosophe procède de même, par remise en cause de l'idée venue spontanément sous la plume, soit à l'instant, soit des années

381 Montaigne, Essais, II, 12, Imprimerie nationale, 1998, p. 801 (apparat critique de la p. 380 , ligne 73 , passage correspondant à la p. 568 de l'éd. Villey-Saulnier, PUF 1965, qui n'enregistre pas la variante). - Les références aux Essais seront ici dédoublées : en premier lieu la page de l'I.N., en second lieu, après barre oblique, la page des PUF. 
auparavant, quitte à la confirmer parfois sous forme de ratification expresse ou de prolongement bien assorti. Ce dédoublement critique était-il pour lui un moyen de conjurer les mirages de toute espèce qu'Érasme avait répertoriés longtemps auparavant dans son paradoxal Éloge de la folie - entre autres le mirage des savoirs accrédités ? Il est possible de faire l'essai de cette hypothèse dans la page même qui comporte la rature, où est esquissé un abrégé singulièrement provocant du même paradoxe. Cela nous donnera, au moins sous forme d'énigmes, les repères indispensables pour une excursion vers la dramaturgie de la folie et du dédoublement, dans laquelle le plus génial des lecteurs de Montaigne a inscrit les fantasmagories autrement troublantes du Roi Lear et de ses compagnons d'infortune.

Le propos en question, inscrit après 1588 sur l'Exemplaire de Bordeaux, prend place dans une méditation sur le rôle des «passions » dans les comportements les mieux délibérés, qui s'achevait sur une consigne de défiance :

Si notre jugement est en main à la maladie même et à la perturbation, si c'est de la folie et de la témérité qu'il est tenu de recevoir l'impression des choses, quelle sûreté pouvons-nous attendre de lui ${ }^{382}$ ?

L'addition transforme d'abord la critique en un éloge inattendu :

N'y a-il point de la hardiesse à la philosophie, d'estimer des hommes qu'ils produisent leurs plus grands effets et plus approchants de la divinité quand ils sont hors d'eux, furieux et insensés? [...] Jamais plus volontiers je ne l'en crus. C'est un pur enthousiasme que la sainte vérité a inspiré en l'esprit philosophique, qui lui arrache contre sa proposition: que l'état tranquille de notre âme, l'état rassis, l'état plus sain que la philosophie lui puisse acquérir, n'est pas son meilleur état. Notre veillée est plus endormie que le dormir, notre sagesse moins sage que la folie. Nos songes valent mieux que nos discours. La pire place que nous puissions prendre, c'est en nous.

On reconnaît sans peine l'allusion aux néo-platoniciens de la Renaissance célébrant les quatre «fureurs", extases prophétiques, poétiques, divinatrices ou amoureuses; ou encore au trop célèbre chapitre XXX des Problemata faussement attribués à Aristote, sur les

\footnotetext{
382 P. 379 / p. 568 . Est citée ici la version de 1588 ; les éditions antérieures donnent : «Si notre jugement est en main à la fausseté même et à l'erreur, si c'est de la folie et de la mensonge qu'il est tenu $[\ldots] »$.
} 
rapports entre la «mélancolie» et le génie. Mais après le bref épilogue sur la «place» que dédaignent les spiritualistes de haute volée, déjà propre à éveiller les soupçons d'un lecteur qui n'a pas oublié que, selon le même Montaigne, «de nos maladies la plus sauvage, c'est mépriser notre être ${ }^{383}$, cette philosophie en mal de vaticinations est réfutée par une réplique sans appel :

Mais pense-elle pas que nous ayons l'avisement de remarquer que la voix qui fait l'esprit, quand il est dépris de l'homme, si clairvoyant, si grand, si parfait, et pendant qu'il est en l'homme, si terrestre, ignorant et ténébreux, c'est une voix partant de l'esprit qui est partie de l'homme terrestre, ignorant et ténébreux: Et à cette cause voix infiable et in croyable 384 .

Faute d'être lui-même extatique, le discours qui fait de l'extase une condition nécessaire de l'accès à la vérité se dénonce comme radicalement erroné ; CQFD. L'éloge qui précède était donc un piège tendu aux érudits férus de mystères, pour se moquer d'eux, en prélude à ce qui esquisse finalement, en forçant un peu le trait, une critique de la folie pure par réflexion sur les cautions dont on la pare... « Tu te joues souvent», se reproche ailleurs l'essayiste, « on estimera que tu dies à droit ce que tu dis à feinte $»^{385}$ : il ne se trompait pas, quelques commentateurs des plus huppés l'ont prouvé naguère à leurs dépens. Ici pourtant leur bévue, si lourde qu'elle soit, peut s'excuser. Montaigne a bien cité sans ironie apparente, à la fin de son chapitre «De l'ivrognerie", les mêmes théories d'Aristote et de Platon sur les rapports entre folie et inspiration; et au sujet de son florilège à la louange «du jeune Caton» il a décrit sur le modèle platonicien l'exaltation que lui fait éprouver «la bonne, l'excessive, la divine» poésie, comme par contagion de «l'inspiration sacrée des muses » ${ }^{386}$. Le traquenard du pseudo-éloge analysé ci-dessus serait-il double, pour ridiculiser symétriquement les enthousiasmes des théoriciens et les réticences des sceptiques - ou pour les mettre en question les uns par les autres? Ainsi se dessinerait un de ces montages paradoxaux, fréquents dans les Essais, qui avivent ou approfondissent en apories des problèmes que l'on croyait faciles à résoudre.

\footnotetext{
383 III, 13, p. 495 / p. 1110 (version de 1588 : « [...] c'est haïr et dédaigner notre être »).

384 II, 12 , p. $380 /$ p. 568 .

385 III, 5, p. 148 / p. 875 (la phrase est une addition de l'E.B.)

386 Dans l'ordre, II, 2, p. 37 / p. 347-348, et I, 37, p. 380 / p. 231-232.
} 
Telles sont les chausse-trapes de la logique. Pour y voir un peu plus clair, reste à recourir au dramaturge qui a su représenter, par confrontation de rôles, les diverses figures de la folie, de ses leurres, et des éclairages qu'elle projette autour d'elle, pour peu qu'elle soit réfléchie en une pensée lucide.

KING LEAR: le titre même attire l'attention sur le vieux roi, et la tradition critique a pu légitimement s'en autoriser pour mettre l'accent sur la démence de celui-ci, dont les phases successives, du ressentiment atrabilaire poussé par degrés au paroxysme de fureur jusqu'à l'égarement puis à la stupeur accablée, articulent l'ensemble de la pièce. La lecture en est facilitée par des modèles culturels bien répertoriés, propres à la magnifier mais surtout à la rendre intelligible. On reconnaît volontiers dans son déchaînement, en rapport symbolique avec la tempête sur la lande, les éclairs et les ténèbres, la débâcle des représentations peinte par Bosch ou Bruegel et décrite par Michel Foucault comme «le grand sabbat de la nature: [...] l'avènement d'une nuit où s'engloutit la vieille raison du monde » ${ }^{387}$. Ses motifs et ses thèmes, en revanche, illustreraient plutôt les méticuleuses analyses de Robert Burton: la détresse, la déception devant l'ingratitude, le dépit, les ressentiments, les remords, tout cela aggravé par l'âge et la faiblesse physique, autant de composantes distinguées et expliquées méthodiquement dans l'Anatomy of Melancholy ${ }^{388}$. Enfin, il est possible d'y déceler les étapes d'un itinéraire spirituel coupé de crises convulsives, depuis l'erreur initiale, le regret et la rage, vers la dépossession et le renoncement. La pièce donne à voir ces divers aspects du désarroi, sans les rendre vraiment déconcertants: ils ne font qu'exposer avec une force extrême la situation du roi déchu.

Mais Lear n'est pas seul. Ses égarements sont confrontés avec la «folie» comique et incisive de son bouffon, the Fool qui l'accompagne jusqu'à la scène vi du troisième acte, et avec la folie que simule Edgar sous les guenilles du poor Tom of Bedlam. Le spectateur est donc en présence de trois langages de la folie, dont les différences et les recoupements partiels sont significatifs, et particulièrement pertinents

${ }^{387}$ M. Foucault, Histoire de la folie à l'âge classique, I, 1, Gallimard (NRF) 1972, p. 32.

388 R. Burton, Anatomy of Melancholy, Première partie, notamment, II, I, 5 et II, III, 4, 6, 9, 10 . 
à la question qui nous occupe puisqu'ils procèdent des diverses attitudes des personnages devant l'énigme de la déraison.

De ces attitudes, celle du Fool est bien connue. C'est le persiflage véridique: à l'origine, le rôle dévolu au bouffon, seul personnage de la cour qui soit exempt des obligations de respect en vertu de son irresponsabilité d'aliéné (ou plutôt de prétendu aliéné ; car lui aussi est un trompe-l'œil, à sa place dans la mascarade du pouvoir). Son discours est censé dissiper les faux-semblants disposés en miroirs et en pièges autour des princes. Il manifeste un parti pris de dépréciation, parfaitement illustré au Moyen Âge par les dialogues de «Salomon » avec son fou Marcoul qui transpose les graves maximes du maître de sagesse en recettes goguenardes ou triviales. Ici, la tâche est à moitié faite : les prestiges de la grandeur et de la puissance se sont vite dissipés autour du vieux roi en butte à l'insolence du premier Oswald venu. Reste à traduire en railleries les frustrations de Lear, comme pour lui faire prendre conscience de l'impuissance à laquelle il s'est aveuglément réduit: A bitter fool! dit le roi ${ }^{389}$. Mais peu auparavant, répondant à Kent déguisé en vagabond qui s'est déclaré as poor as the king, il a lui-même constaté sa situation : If thou be'st as poor for a subject as he is for a king, thou art poor enough ${ }^{390}$. Dans ces conditions, le Fou ne révèle rien; il se borne à transposer sur son registre sarcastique la partie de la tragédie au cours de laquelle le sang n'a pas encore coulé ; après quoi il disparaît, en exprimant en termes d'horaire quotidien l'énigme du monde à l'envers :

LEAR. [...] We'll go to supper i'the morning. So, so, so.

FOOL. And I'll go to bed at noon 391 .

Bien différente est la folie d'Edgar, ou plutôt celle qu'il simule, his sullen and assumed humor of Tom of Bedlam ${ }^{392}:$ d'entrée de jeu,

\footnotetext{
389 «Bien amer, ce bouffon », King Lear, I.iv.114 (les références des textes de Shakespeare renvoient à l'édition de Camille Chemin, Paris, Aubier, 1942, corrigée par endroits d'après The complete works of W. Shakespeare, Spring Books, London, s.d. [1964]).

390 «Si tu es aussi pauvre comme sujet qu'il l'est comme roi, tu es bien pauvre» (I.iv.18-9).

${ }^{391}$ LEAR - Nous irons souper demain matin. C'est ça, c'est ça...-LE Fou - Et moi, j'irai au lit à midi. (III.vi.37 — George Hoffmann me suggère que le moment assigné à cette disparition fait du Fool une ombre, réduite à rien à l'heure où le soleil est au zénith; ce qui s'accorde avec la réplique célèbre qui, à la faveur d'une équivoque syntaxique, tout à la fois identifie le Fool et définit le statut de son maître : Lear's shadow (I.iv.187).
} 
c'est une fantasmagorie démoniaque. Il ne joue pas le fool, mais le madman (III.iv.41sd), que le bouffon prend d'abord pour un spirit, et qui balbutie (grumbles) à son entrée en scène: Away! the foul fiend follows $m e^{393}$. L'apparition est assez effrayante pour que le Fou, comme épouvanté par sa chanson du Pillicock et ses hululements, craigne d'être gagné par la démence: This cold night will turn us all to fools and madmen ${ }^{394}$. De fait, les paroles d'Edgar ont des relents de hantise infernale, que ses aveux stéréotypés de coquetterie, de paillardise, de mensonge etc., plus loin, ne suffisent pas à moraliser. Leur imagerie relève de l'hallucination, effet ou matière, selon R. Burton $^{395}$ des tourments que subit le possédé,

... whom the foul fiend hath led through fire and through flame, through ford and whirlpool, o'er bog and quagmire ; that hath laid knives under his pillow, and halters in his pews ; set ratsbane by his porridge ${ }^{396}$..

Et plus loin, les nourritures dont il se repaît, des grenouilles aux charognes, évoquent, par-delà le dégoût, les interdits alimentaires de la Bible : à leur contact, il est impur,

Poor Tom : that eats the swimming frogs, the toad, the tadpole, [...] that in the fury of his heart, when the foul fiend rages, eats cow-dung for sallets ; swallows old rats and the ditch- $\operatorname{dog}^{397} \ldots$

Après quoi surgissent les diables, distinctement suscités par leurs noms d'Enfer :

Beware my follower. Peace, Smulkin, peace, thou fiend ! [...]

The prince of darkness is a gentleman :

Mode he's call'd, and Mahu. ${ }^{398}$

\footnotetext{
392 «sa sombre humeur factice de fou de Bedlam» (présentation de l'édition de 1608, d'après la notice de B. Hodek, Spring Books, p. xx).

393 «Arrière! L'esprit immonde me poursuit» (III.iv.42).

394 «Cette nuit glacée fera de nous tous des fous et des déments » (III.iv.69).

395 Op. cit., Première partie, II, I, 2 : les esprits du feu « entraînent les hommes dans les rivières ou dans les précipices", d'autres esprits font surgir des flammes, jettent des pierres, font cliqueter des chaînes, secouent les hommes...(Burton emprunte de tels détails à Bodin et à Cardan).

396 «...que l'esprit immonde a chassé à travers feu et flamme, gués et remous, bourbiers et fondrières; il a mis des couteaux sous son oreiller, des cordes de pendus sur son prie-Dieu, de la mort-aux-rats au bord de sa bouillie...» (III.iv.47-50).

397 «Pauvre Tom, qui mange la grenouille dans l'eau, le crapaud, le têtard [...] qui dans sa furie, quand l'esprit immonde se déchaîne, mange de la bouse de vache en guise de salade, avale vieux rats et chiens crevés » (III.iv.108-10).
} 
On comprend dès lors la réplique de Lear à Gloucester qui voudrait l'entraîner à l'écart :

First let me talk with this philosopher.

What is the cause of thunder ? ${ }^{399}$

À ses yeux, l'énergumène est "philosophe », dépositaire d'un savoir secret, en rapport avec les perturbations célestes comme la foudre où l'on déchiffre des signes de la colère de Dieu. Le délire du pauvre Tom indique ainsi le sens de toute la scène de la tempête, foul weather qui se déchaîne autour de Lear $^{400}$, assorti au foul fiend qui persécute l’insensé.

Seulement, cette folie dotée de ses plus mystérieuses résonances démoniaques et cosmologiques est entièrement simulée. Le spectateur est censé le savoir dès l'entrée en scène d'Edgar, à en juger par la rubrique qui précise, avec le vrai nom de celui-ci, la tenue à travers laquelle on doit le reconnaître : Enter Edgar, disguised as a madman. Et surtout, la simulation est représentée comme au second degré dans la scène vi de l'acte III, où est joué le procès des filles de Lear. Le passage mérite un examen assez attentif, car il produit d'étranges interférences entre les trois « folies ».

Dès qu'est installé le tribunal imaginaire, Edgar entre dans ce délire du roi. Lorsqu'il entend celui-ci convoquer les « renardes», il en interpelle une pour lui faire voir les yeux (de Lear? d'un démon? impossible de trancher) qui la fixent :

LEAR. [...] Now you she-foxes !

EDGAR. Look, where he stands and glares ! Wantest thou eyes at trial, Madam ${ }^{401}$ ?

\footnotetext{
398 « Prenez garde à qui me suit. Paix, Smulkin, paix, démon ! [...] Le prince des ténèbres est gentilhomme : il s'appelle Mod, et Mahu » (III.iv.117).

399 «Laissez-moi d'abord parler avec ce philosophe... Quelle est la cause du tonnerre?» (III.iv.130-1)

${ }^{400}$ Kent. Who is there, besides foul weather? - THE GENTLEMAN. One minded like the weather, most unquietly (III.i.1-3 : «Qui est là, outre ce maudit temps ? - Un dont l'esprit est comme le temps, en plein dérangement». Voir aussi III.iv.12, The tempest in $m y$

mind...).
${ }^{401}$ L. - Maintenant, à vous, renardes ! - E. - Voyez, il se tient là, le regard fixe... Veux-tu des yeux pour ton procès, Madame? (III.vi.21-24).
} 
Mais il partage aussi les bouffonneries du Fou, en chantonnant avec lui :

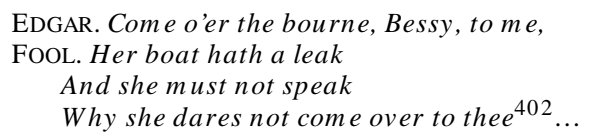

quitte à réintégrer la chanson à deux voix dans son pseudo-délire de possédé :

EDGAR. The foul fiend haunts poor Tom in the voice of a nightingale. Hopdance cries in Tom belly for two white herrings. Croak not, black angel, I have no food for thee ${ }^{403}$.

Puis chansons et bouffonneries sont à leur tour incorporées à la procédure imaginaire; à la formule quasi-juridique d'équité par laquelle il ouvre la séance Edgar enchaîne un refrain de pastourelle, qu'il interrompt d'une apostrophe à quelque démon ; et le Fou dénonce comiquement la mise en scène :

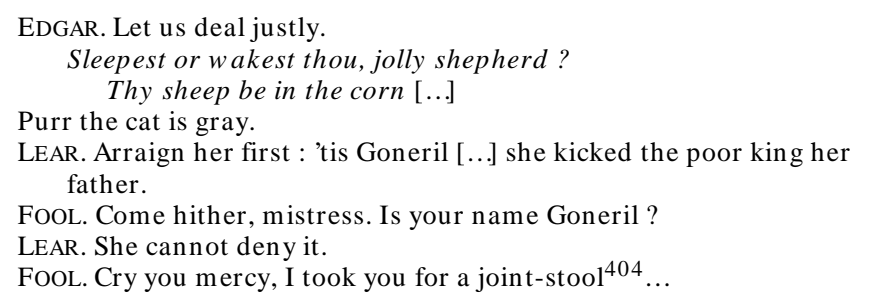

Le mélange des fantasmagories continue après la crise de fureur hallucinée de Lear, qui finit par donner à Edgar le thème d'un

\footnotetext{
402 E. - Passe le ruisseau, Betsy, viens à moi. - F. - Sa barque a une fissure, et elle ne doit pas dire pourquoi elle n'ose pas aller à toi. (ibid.)

403 «L'esprit immonde hante le pauvre Tom avec une voix de rossignol. Hopdance crie dans le ventre de Tom, pour deux harengs blancs. Ne croasse pas, ange noir, je n'ai pas de nourriture pour toi. » (III.vi.29-32 - Merci à Jean-Marie Maguin d'avoir indiqué au cours de la discussion que l'acteur qui jouait le rôle du Fou, et peut-être aussi celui de Cordelia, était réputé pour ses talents de chanteur ; d'où la « voix de rossignol »).

404 E. - Procédons avec justice. Dors-tu ou veilles-tu, joyeux berger? Tes brebis sont dans les blés [...] Pur le chat est gris. - L - - Mettez d'abord celle-ci en accusation, c'est Goneril [...] elle a bousculé d'un coup de pied le pauvre roi, son père. - F. - Venez ici, Mademoiselle. Votre nom est Goneril ? - L. - Elle ne peut pas le nier. - F. - Je demande pardon, je vous prenais pour un tabouret. (III.vi.40-51 — «Pur» est le nom d'un démon mentionné par Harsnett dans sa Declaration of egregious Popish Impostures).
} 
exorcisme délirant, geste d'automutilation et couplet apotropaïque en assonances :

LEAR. The little dogs and all,

Tray, Blanche and Sweet-heart, see, they bark at me.

EDGAR. Tom will throw his head at them.

Avaunt, you curs!

Be thy mouth or black or white,

Tooth that poisons if it bite,

Mastiff, greyhound, mongrel grim [...]

Tom will make them weep and wail:

For with throwing thus my head,

Dogs leap the hatch and all are fled ${ }^{405} \ldots$

Folie du vieillard, bouffonneries et simulations de ses acolytes se combinent dans le procès des filles coupables, et elles le réduisent à ce qu'il est : un spectacle de démence collective, en attente de sens.

Car en fait, elles fournissent à ce procès toute sa matière. Jusqu’à présent, le spectateur n'a constaté, en fait de crimes, que les perfidies d'Edmond (ici hors jeu) et les manœuvres provocatrices de Goneril et de Regane qui donnent lieu à la fureur insensée de leur père. C'est sur le théâtre d'ombres que les gestes de celles-ci sont amplifiés, à la fois par le cortège diabolique du poor Tom qui donne leurs résonances d'outre-tombe aux fantasmes d'enfer de Lear, et, en contraste, par le persiflage du Fool qui réduit tout à une sinistre farce. Peu après (III.vii), le sang va couler des orbites lacérées de Gloucester, de la blessure de Cornouailles, de son serviteur tué par Goneril; suivront d'autres atrocités. Pour l'instant, tout est encore virtuel. En somme, ce qui légitimerait la fureur de Lear et lui donne un sens est joué à vide par les pseudo-fous autour de lui, qui manipulent, à son initiative, des figures de hantises absentes avec leurs dérisoires supports (un tabouret, un lieu de gesticulations en guise de prétoire...), à distance de critique ou d'effarement. En résulte, de plus, une disproportion saisissante entre la faute et les sanctions imaginaires, qui s'égarent dans l'horreur: fourches chauffées au rouge pour supplicier les coupables, dissection de Regane pour examiner la gangue qui a durci son cœur...En ce point mieux qu'en aucun autre, la pièce est une tragédie de la folie. Et lorsque le poids des vrais malheurs commence à

405 L. - Les petits chiens, tous, Tray, Blanche, Doucette, regardez, ils aboient contre moi. E. - Tom va leur jeter sa tête. Au large, maudits roquets! Museau noir ou museau blanc, Morsure et venin de dent, Mâtin, lévrier, bâtard [...] Tom fera gémir, hurler, En jetant sa tête en l'air, Sauter clos et tous filer... (III.vi.18-28). 
écraser les protagonistes, le même éclairage confère à la réalité des traits de cauchemar. Gloucester et Lear découvrent l'un après l'autre qu'ils ont été objets de divertissement cruel et de spectacle pour les dieux :

GLOUCESTER. As flies to wanton boys, are we to the Gods, They kill us for their sport...

LEAR. When we are born, we cry that we are come To this great stage of fools 406 .

Ainsi est décelé dans leur détresse l'archétype de ce qu'Edgar a donné à voir tant qu'il a incarné le poor Tom, un jeu insensé dont le concepteur est à la fois metteur en scène, victime et comédien bouleversé par son rôle :

Bad is the trade that must play fool to sorrow,

Angering itself and others 407 .

Est-ce à dire que la pièce que jouent ces fools est totalement étrangère au comique? Laissons la question en suspens ${ }^{408}$, non sans penser aux bouffonneries grinçantes de Beckett, quelques siècles plus tard, ou de Taborit. Il importe surtout de mettre l'accent sur l'effet scénique qui introduit dans le délire des personnages le regard imperceptiblement distancié du spectateur qui «cherch[e] avidement [à] reconnaître en ombre même et en la fable des théâtres, la montre des jeux tragiques de l'humaine fortune ${ }^{409}$

406 G. - Comme des mouches aux mains d'enfants capricieux, ainsi sommes-nous aux mains des dieux : ils nous tuent pour s'amuser. (IV.i.36-7) - L. - En naissant, nous pleurons d'entrer en scène sur ce grand tréteau de fous. (IV.vi.180-1 — la leçon est adressée à Gloucester).

407 E. - Triste métier, d'avoir à jouer le fou devant le chagrin, en irritant sa douleur avec celle d'autrui. (IV.i.38-9, en aparté après la sentence de Gloucester citée ci-dessus).

$408 \mathrm{Au}$ cours de la discussion, J.-M. Maguin a rappelé que jusqu'en 1950 il était traditionnel de couper, à la représentation, la scène du procès imaginaire, parce que, disaiton, elle risquait de provoquer des rires intempestifs. Le prétexte était spécieux : lorsque Peter Brook a réintroduit cette scène, elle n'a pas diminué la puissance tragique de l'ensemble, bien au contraire. En fait, elle faisait peur, justement parce qu'elle troublait la répartition des fonctions théâtrales. En l'éliminant, ou en la jouant sur un registre uniformément grave, si c'était possible, on éviterait au spectateur l'angoisse et le malaise, variante du phobos kai eleos des tragiques grecs, que la dramaturgie shakespearienne, ici plus qu'ailleurs, tend à exacerber.

409 Essais, III, 12, p. 397 / p. 1046. 
Cette dernière expression nous a ramenés à Montaigne. Il l'a inscrite sur l'Exemplaire de Bordeaux en marge d'une évocation précise des guerres civiles et de la peste qui venaient de ravager la Guyenne en 1586, tragédies bien réelles qui l'avaient mis en extrême danger, lui et sa famille, et qui pouvaient se reproduire à tout moment. Il n'oublie pas ces scènes de mort, peut-être même l'obsèdent-elles de «malplaisantes pensées », comme il l'a écrit un peu plus haut; mais il les assimile aux fictions théâtrales en prenant en compte le regard qu'il pose sur elles, en contrechamp. C'est là l'essentiel : une sorte de retrait ironique, d'examen qui met à distance menaces et fantasmes, rend possible l'essai d'une pensée consciente de ses risques d'aberration, et toujours en quête de lucidité. Ainsi peuvent être démentis les leurres de la folie pure, prétendues inspirations ou révélations qui falsifient les «jeux tragiques de l'humaine fortune», les travestissant en cauchemars de fin du monde ou les édulcorant en légende dorée. Lear donne l'exemple de ces deux types d'égarement: du premier, presque tout au long de la pièce ; du second, à l'instant d'illusion où il s'avance avec Cordelia vers une prison qu'il croit devoir partager avec elle,

so we'll live,

And pray, and sing, and tell old tales, and laugh

At gilded butterfly [...]

And take upon's the mystery of things,

As if we were God's spies 410 .

Edgar, si grave et douloureuse que soit son ironie, incarne au contraire le dédoublement. Non seulement il se déguise en madman, mais lorsque ce déguisement n'a plus de motif il continue à dissimuler son identité. C'est ainsi qu'il s'abstient, étrangement, de se faire connaître à son père aveugle, alors qu'il vient d'entendre le souhait de celui-ci : toucher encore une fois au moins le visage de son fils maudit par erreur. Il doit faire effort pour garder le masque : 410 L. - Nous passerons la vie ainsi, à prier, à chanter, à nous dire de vieux contes, à rire aux
papillons dorés [...]; nous prendrons en charge le mystère des choses, comme si nous étions les espions de Dieu. (King Lear, v.iii.11-17). 
EDGAR. Poor Tom a-cold. (aside) I cannot daub it further

GLOUCESTER. Come hither, fellow.

EDGAR. (aside) And yet I must ${ }^{411}$.

— Mais pourquoi «doit»-il le garder? Plus tard, au moment du simulacre de chute censé détourner l'aveugle de son projet de suicide, il entend Gloucester regretter sa malédiction et prier pour lui : If Edgar live, $O$, bless him ${ }^{412}$ ! Pourtant, lorsqu'il le relève, il prend l'attitude d'un simple passant et décrit même comme une sorte de démon le personnage qu'il jouait auparavant. Plus loin, devant Oswald qu'il va tuer, il se mue en rustre patoisant : pourquoi ? Enfin, au dernier acte, lorsqu'il évoque devant Albany les ultimes instants de son père, il se reproche amèrement de ne pas s'être fait connaître plus tôt :

Never - O fault ! - reveal'd myself unto him, until some half-hour past, when I was arm'd ${ }^{413}$

C'est dire à demi-mot que la dissimulation lui était imposée par autre chose que le devoir ou le sentiment, par rapport auxquels elle est une «faute».

Elle n'est vraiment requise en effet que par son rôle dans la configuration logique de la pièce. Edgar est celui qui se dédouble, celui dont l'identité ne va pas de soi : le sujet ironique, reflet inversé du frénétique Lear. Le roi est fasciné jusqu'à l'aliénation par la fantasmagorie de mensonge et de cruauté qui régit this great stage of fools: machinations de Goneril, de Regane et d'Edmund, absurdes malédictions prononcées contre Cordelia, contre Edgar, bannissement de Kent le véridique... Edgar s'affranchit de cette fascination dans la mesure où il l'imite, en la peuplant même de simulacres surgis de l'enfer. Son regard lucide ne réduit pas la folie à néant ; au contraire, il la joue, il y participe sans y croire et parle son langage à la perfection, mieux que les fous, parce qu'il le parle en connaissance de cause, sous

${ }^{411}$ E. - Le pauvre Tom a froid. (à part) Je ne puis feindre plus longtemps. - G. - Approchetoi, mon ami. - E. - (à part) Et pourtant, il le faut. (IV.i.52-3 — le souhait de Gloucester était exprimé dans la même scène (22-3); rien ne dit qu'Edgar ne l'a pas entendu).

412 G. - Si Edgar est vivant, ô ! qu'il soit béni. (IV.vi.40).

413 E. - Jamais - ô quelle faute ! - je ne m'étais fait connaître à lui, jusqu'au moment, il y a une demi-heure, où je fus armé pour le combat (V.iii.183-4). 
un masque d'acteur qu'il ôte dans les moments de solitude pour se muer en spectateur : Tom, aw ay !/ Mark the high noises ${ }^{414}$.

Ce double jeu de la folie critique nous ramène au texte d'où nous sommes partis. Montaigne s'y moquait des théoriciens de l'inspiration qui cautionnent les révélations surnaturelles dont ils rêvent, et avec elles l'influx divin censé les apporter, par les inférences de leur esprit «terrestre, ignorant et ténébreux», sans voir que cette gnoséologie de l'enthousiasme sape ses propres bases, et fait écrouler le système. Sa démonstration est irréfutable. Mais elle ne saurait cautionner, en retour, une pensée strictement rationnelle: l'esprit «terrestre, ignorant et ténébreux » qui a celle-ci en partage ne peut pas sans imposture se prévaloir d'une vérité absolue, même pour déclarer qu'il est «terrestre, ignorant et ténébreux». Autrement dit, le philosophe peut bien se gausser du visionnaire, mais pas pour se prétendre à sa place détenteur du savoir. « Nous voilà au rouet ».

Ou plutôt, le dispositif logique est instable, et doit le rester : la contestation des leurres postule une vérité, mais celle-ci serait un leurre de degré supérieur si elle était assertée en conclusion. Cette torsion caractéristique de l'argumentation pyrrhonienne est à l'image du contexte sur lequel se greffe la pseudo-théorie de l'enthousiasme. Montaigne, revenant sur les assises philosophiques de son «apologie de R. Sebond » qu'il a close par une adresse à la destinataire ${ }^{415}$, vient de mesurer l'instabilité des croyances d'après sa propre expérience, des fluctuations de son esprit selon les «humeurs» qui le sollicitent. Il illustre son propos en décrivant les intermittences du désir, et des représentations qui en procèdent :

Cette passion qu'on dit être produite par l'oisiveté au cœur des jeunes hommes [...] elle représente bien évidemment, à ceux qui ont essayé de s'opposer à son effort, la force de cette conversion et altération que notre jugement souffre. [...] Je la sentais naître, croître et s'augmenter en dépit de ma résistance : [...] je voyais évidemment grossir et croître les avantages du sujet que j'allais désirant, [...] les difficultés de mon

414 E. - Tom, va-t'en. - Observe le grand tintamarre. (III.vi.108-9 - sitôt congédié son personnage de fou, Edgar se place en position d'observateur du «tintamarre» de l'universelle erreur).

415 «Vous, pour qui j'ai pris la peine d'étendre un si long corps...», Essais, II, 12, p. 362 / p. 557. - A partir de cette apostrophe, il n'est plus question de Sebond ; Montaigne expose directement la critique pyrrhonienne de la connaissance, qui sous-tendait son argumentation paradoxale pour et contre le théologien. 
entreprise, s'aiser et se planir [...] : mais ce feu étant évaporé [...], mon âme reprendre une autre sorte de vue, autre état et autre jugement : les difficultés de la retraite me sembler grandes et invincibles, et les mêmes choses de bien autre goût et visage que la chaleur du désir ne me les avait représentées. Lequel plus véritablement, Pyrrhon n'en sait rien ${ }^{416}$.

L'exemple est classique, mais son épilogue ne l'est pas. Tous les moralistes, depuis l'Antiquité, ont dénoncé les mirages du désir, mais l'usage est de présenter le désenchantement, quelle qu'en soit la cause, comme un retour au réel, authentifié par sa banalité même. Montaigne reprend à son compte la critique des illusions, mais lui associe la critique symétrique, des désillusions : pourquoi ces dernières seraientelle plus véridiques? On peut constater que le regard a changé, c'est tout ; rien ne dit qu'il n'est pas, dans les deux cas, tributaire d'humeurs parfaitement irrationnelles: «des effets d'une passion ardente, nous retombons aux effets d'une passion frileuse».

Peut-être en va-t-il de même pour la méditation sur les pouvoirs de la folie. Le texte imite de toute évidence l'exaltation progressive des fervents de l'inspiration, et jusqu'au choix préjudiciel qui la commande : la philosophie avoue-t-elle son infériorité ? «jamais plus volontiers je ne l'en crus »; et la «sainte vérité » profite de cette bonne volonté muée en «pur enthousiasme» pour dicter des paradoxes en style oraculaire, avec anaphores et chiasme d'oxymores réitérés. Après quoi, des procédés stylistiques tout aussi insistants marquent l'artifice opposé, d'une dialectique de réfutation aux arguments un peu trop bien ajustés. «Lequel plus véritablement, Pyrrhon n'en sait rien». Le jeu indique la seule échappatoire plausible : faire valoir comme son objet essentiel le contrôle réflexif de ces idées et de leur panoplie sophistique. C'est là une variante de la khrêsis phantasiôn («gestion des représentations ») préconisée par Épictète : le tout est de prendre les «fantasies» de l'esprit ou du cœur pour ce qu'elles sont — des «fantasies», précisément, tributaires de toutes sortes d'influences contingentes, qui peuvent être avouées, assumées, ratifiées par le jugement, mais non pas travesties en vérités absolues. Et si toute erreur est « folie », si toute errance est « vanité », reste au «badin de la farce », pour vocation, la critique vigilante de sa propre folie ; grâce à quoi il pourra s'appliquer à «faire bien l'homme et dûment», en jouant correctement le rôle qu'il assume on this stage of fools.

${ }^{416}$ P. $381 /$ p. 569 , ainsi que la citation suivante. 
Mais c'est dans la tragédie de Shakespeare que l'on distingue le plus clairement la nécessité de cette critique de la folie pure, de cet examen lucide, à distance, des convulsions des hommes dans « le bruit et la fureur », avec pour dernier recours l'ironie du désespoir dont la formule est donnée, comme de juste, par Edgar :

The lowest and most dejected thing of fortune

Stands still in esperance, lives not in fear :

The lamentable change is from the best;

The worst turns to laughter 417 .

C'est là aussi qu'elle atteint ses limites : là où se profile le mal en ce qu'il a d'inexprimable, lorsque la raison et la lucidité perdent leurs droits - lorsque Cordelia est morte. Elle avait dit la vraie souffrance, celle que l'on éprouve à voir s'effondrer l'être aimé: For thee, oppressed king, am I cast down ${ }^{418}$. À la fin de la dernière scène, Lear entre portant dans les bras son cadavre. Nul n'arrive à qualifier ce paroxysme de détresse :

KENT. Is this the promised end?

EDGAR. Or image of that horror?

ALBANY. Fall, and cease ${ }^{419}$

et l'égarement du vieux roi est seul à la mesure du scandale absolu ${ }^{420}$. Les témoins en sont réduits à ne plus dire que leur accablement :

${ }^{417}$ E. - Au plus profond des disgrâces de la fortune, on demeure dans l'espérance, on ne vit plus dans la peur : le changement qui afflige, c'est celui qui dépossède du bien ; au plus mal, revient le rire. (IV.i.3-6, mais voir la note suivante. - Cf. Montaigne : « Tout ainsi que des chemins j'en évite volontiers les côtés pendants et glissants, et me jette dans le battu le plus boueux $[\ldots]$ : Aussi j'aime les malheurs tout purs, qui ne m'exercent et tracassent plus après l'incertitude de leur rhabillage, et qui, du premier coup, me poussent droitement en la souffrance [...] La plus basse marche est la plus ferme : C'est le siège de la constance.» Essais, II, 17, p. $501 /$ p. 644-645).

418 C. - C'est pour toi, roi accablé, que je m'afflige. (v.iii.5. - Edgar de même, juste après le monologue cité ci-dessus, avait découvert en voyant son père en détresse un surcroît de malheur auquel il ne s'attendait plus : "O gods, who is't can say: I am at the worst ? I'm worse than e'er I was », IV.i.24-6)

${ }^{419}$ K. - Est-ce la Fin prophétisée ? -E. - Ou une image de cette horreur ? - A. - Tomber, cesser! (V.iii.238-9)

${ }^{420}$ Le début de sa dernière réplique pourrait le marquer par un étrange détour d'expression, emprunté à Montaigne. Celui-ci, dans le chapitre «De la tristesse » (Essais, I, 2), avait commenté l'exemple de Psammenite silencieux devant les pires sujets de deuil (sa fille en esclavage, son fils mis à mort) mais s'affligeant sur un domestique captif : «ce seul déplaisir se peut signifier par larmes, les deux premiers surpassant de bien loin tout moyen de se pouvoir exprimer» (p.54/ p. 12). Lear au sortir de sa stupeur (notée par Albany: « He knows not what he says», v.iii.268) dit: "And my poor fool is hang'd » (280). 
EDGAR. The weight of this sad time we must obey

Speak what we feel, not what we ought to say ${ }^{421}$.

- ce qui revient à se taire, comme Hamlet: The rest is silence. Montaigne n'a pas dirigé son regard vers ces ténèbres. Ou bien peutêtre a-t-il gardé le silence.

André TOURNON

Université d'Aix-Marseille I

L'expression désigne-t-elle Cordelia («ma pauvre sotte», terme affectueux ? on le verrait plutôt employé dans une interpellation) ou, à la lettre, le Fool? Dans cette seconde hypothèse (corroborée par la conjonction «And», qui relierait le décès du bouffon à ceux qui viennent d'être mentionnés) l'incongruité apparente serait comme chez Montaigne le signe de l'indicible, de même que les brisures des derniers mots : « Do you see this ? Look on her...look...her lips...look there...look there».

${ }^{421}$ E. - Au poids de ce triste temps il nous faut obéir : exprimer ce que nous ressentons, non ce qu'il faudrait dire (v.iii.298-9. — Ces mots sont attribués à Albany dans le Quarto (et dans l'éd. de référence), à Edgar dans le Folio. La distribution des répliques paraît confirmer la seconde version : Albany vient de solliciter l'assistance de Kent et d'Edgar pour gouverner l'Angleterre ; Kent s'est désisté, en faisant comprendre qu'il va suivre Lear dans la mort ; reste à entendre la réponse d'Edgar). 